\title{
Low-grade fever: how to distinguish organic from non-organic forms
}

\author{
M. Affronti, P. Mansueto, M. Soresi, A. M. Abbene, A. Affronti, M. Valenti, L. Giannitrapani, \\ G. Montalto
}

Department of Clinical Medicine and Emerging Pathologies, University of Palermo, Palermo, Italy

Correspondence to: Prof. Giuseppe Montalto, Dipartimento di Medicina Clinica e delle Patologie Emergenti, Università di Palermo, via del Vespro 141, 90127 Palermo, Italy Tel.: + 390916552991 Fax: + 390916552847 Email: gmontal@unipa.it

\section{Disclosures}

The authors declare that the work is original, that they all meet the criteria for authorship, including acceptance of responsibility for the scientific content of the manuscript, that they have no conflict of interest and that this work did not have financial support.

\section{SUMMARY}

Background and aim: Low-grade fever (LGF) is defined as a body temperature between 37.5 and $38.3{ }^{\circ} \mathrm{C}$, which is below the classical value reported for fever of unknown origin (FUO). We attempted to characterise its epidemiology, aetiology and clinical aspects to improve the methodological approach to diagnosis. Design and Methods: We reviewed and evaluated a survey of patients with LGF, followed as outpatients of our Department, a tertiary referral centre from 1997 to 2008. The same classifications were applied for classical FUO, and in the patients diagnosed with LGF, we also investigated for habitual hyperthermia $(\mathrm{HH})$. Results: Seventy-three patients were selected and divided into two groups: group A included 32 patients classified with organic fever and group B included 41 patients with $\mathrm{HH}$. Aetiology of organic LGF was: infectious disease $59 \%$; neoplasm 3.1\%; inflammatory non-infectious disease 6.2\%; miscellaneous $18.7 \%$; undiagnosed $12.5 \%$. Mean age was significantly higher in the organic fever than in the $\mathrm{HH}$ group $(\mathrm{p}<0.02)$. Splenomegaly and loss of weight were significantly associated with organic fever $(p<0.05)$, while dizziness and general malaise were associated with $\mathrm{HH}$. Lack of any pathological signs at physical examination was significantly more frequent in $\mathrm{HH}(p<0.0001)$. Among the biochemical tests, white blood cells and $\mathrm{C}$-reactive protein were more frequently above normal limits in group $A$ than in group $B(p<0.05)$. Conclusions: In our experience, LGF requires the same methodological diagnostic approach as FUO, because there is no relationship between body temperature values and the severity of the underlying diseases, and the aetiological spectrum is also the same.

\section{Introduction}

Fever is one of the most common clinical manifestations referred by patients to their physicians (1). The challenge is to distinguish between fevers caused by the more or less serious pathologies, requiring a specific therapy, and those caused by the vast majority of other ailments, which instead often present a self-limited pathology. Fever is defined as an increase in body temperature mediated by a functional alteration of the regulatory centre of the hypothalamus, causing a rise in temperature towards the upper values of the set-point, the activation of the peripheral mechanisms of thermogenesis and the inhibition of those of thermodispersion $(2,3)$. Hyperthermia, on the contrary, is an increase in body temperature independent of the physiological homeostatic control mechanisms, which do not, however, raise the hypothalamus setpoint. In other words, it arises from a 'peripheral' alteration of the mechanisms of thermoproduction and thermodispersion.

Another important condition is the fever of unknown origin (FUO), which poses considerable problems for physicians, because although most diseases underlying FUO are treatable, they can be difficult to diagnose in a particular patient and for reasons which are not always clear (4-11).

Low-grade fever (LGF) commonly refers to a condition with a body temperature continually or intermittently between 37.5 and $38.3{ }^{\circ} \mathrm{C}$. As in the case of fever, it is absolutely a symptom accompanying very many infectious, and autoimmune and neoplastic diseases. Sometimes, however, there is no particular organic pathology, as in the case of habitual hyperthermia $(\mathrm{HH})$, which, rather than a disease, should 
be considered a paraphysiological variant of normal body temperature (6).

Habitual hyperthermia is a clinical condition characterised by a body temperature never higher than $38.3^{\circ} \mathrm{C}$, with an erratic circadian rhythm. It may persist for years and for rather complex reasons, and the normal body temperature of an otherwise perfectly healthy subject remains elevated. It is typical of young asthenic women prone to headaches and with vasomotor liability. Its diagnosis today is still possible, but only after an adequately prolonged period of observation and measurement of body temperature (6). Although FUO is widely recognised and is frequently reported, in our opinion, LGF has not received adequate attention in the literature. This work, therefore, reviews our clinical experience of patients with LGF, with the aim of shedding further light on its frequency, causes, management, work-up, prognosis and possible links with the much betterknown forms of FUO.

\section{Patients and methods}

Our study group included all the cases referred for LGF between 1997 and 2008 as outpatients at our Department of Clinical Medicine, Policlinico Hospital, which is a tertiary referral centre.

Inclusion criteria were patients with axillary body temperature continually or intermittently between 37.5 and $38.3{ }^{\circ} \mathrm{C}$ for at least 3 weeks. Patients with axillary body temperature $\geq 38.3{ }^{\circ} \mathrm{C}$ at any time were considered to be classical FUO and, therefore, excluded. Patients who presented with LGF with particular symptoms or alarm signs (such as dysphagia, rectorrhagia, severe weight loss, neurological disorders, etc.), suggesting a serious organic disease were also excluded.

We used the same classification that is generally adopted for FUO, distinguishing between the classical diseases (infectious disease, neoplasm, inflammatory non-infectious disease) and diseases that are not easily classifiable (miscellaneous). In patients diagnosed with LGF, we also investigated for $\mathrm{HH}$, a paraphysiological condition not associated with any organic disease (6).

The first diagnostic step was a detailed anamnestic investigation and thorough physical examination. The patients' axillary temperatures were then measured and they were asked to measure it in the same manner four times a day and to record results for 5 days in a special booklet. When the anamnestic and physical examination data indicated a strong hypothesis of organic LGF, further appropriate biochemical and instrumental tests were performed. If history was not indicative and/or physical examination was negative, a strong hypothesis of $\mathrm{HH}$ was made. In this case, only total blood cell count, erythrocyte sedimentation rate, $\mathrm{C}$-reactive protein $(\mathrm{CRP})$ and urine analysis were performed. If these were positive, clinical investigations continued with other examinations, otherwise a bi-monthly follow up for 2 years in the same manner and a continual monitoring of temperature were scheduled.

Statistical analysis was performed using Student's $t$-test, the chi-square test and the Fisher exact test, wherever appropriate. The diagnostic reliability of organic LGF was evaluated by calculating sensitivity, specificity, positive predictive value and negative predictive value, using standard formulas. $\mathrm{p}<0.05$ was considered significant.

\section{Results}

Eighty-two patients were selected and, on the basis of the final diagnosis, divided into two groups: group A included 32 patients (14 men, 18 women) with organic LGF, and group B included 50 subjects with HH. In the latter group, however, five subjects were excluded because they did not complete the planned follow up, and after telephone invitations to attend proved unsuccessful, they were considered to have dropped out. Forty-five patients (16 men, 29 women) were therefore eventually included in this group.

Aetiology of organic LGF (group A) is shown in Table 1. In 19 out of 32 patients (59\%), it was because of underlying infectious disease; one patient (3.1\%) had neoplasm, two $(6.2 \%)$ had inflammatory non-infectious disease and six (18.7\%) had miscellaneous causes. In four patients $(12.5 \%)$, there was no definite diagnosis for organic LGF (undiagnosed LGF): biochemical tests showed only aspecific changes, imaging procedures were not diagnostic, and in any case the fever disappeared within 4 months.

Diagnosis of $\mathrm{HH}$ was hypothesised after the first evaluation, including history and physical examination, in $45 / 77$ patients $(58.5 \%)$. In four patients, however, the initial diagnosis was not confirmed and in the following 6 months these diagnoses were made: one intestinal bacterial contamination in a patient with dietary intolerance; one dental granuloma and one appendicitis with histological features of Crohn's disease; in the fourth case, sinusitis in a subject allergic to pollen with rhinitis was diagnosed after 12 months. A definitive diagnosis of $\mathrm{HH}$ was therefore made in 41 out of 77 patients after 2 years of follow up.

Mean age was significantly higher in the organic LGF group A than in the $\mathrm{HH}$ group B (34 \pm 14 vs. 
Table 1 Final diagnosis of patients with low-grade fever (LGF), diagnostic tools and hypothesised diagnosis at first evaluation

\begin{tabular}{llll}
\hline Diagnosis & Patients $\boldsymbol{n}(\%)$ & Diagnostic tools & First evaluation \\
\hline Mononucleosis & $4(12.5)$ & Serology & Organic LGF \\
Brucellosis & $6(18.8)$ & Serology & Organic LGF \\
Autoimmune thyroiditis & $2(6.3)$ & Autoantibodies/ultrasound scan & Organic LGF \\
Toxoplasmosis & $2(6.3)$ & Serology & Organic LGF \\
Cat-scratch disease & $1(3.1)$ & Serology & Organic LGF \\
Sinusitis & $1(3.1)$ & Radiography & Habitual hypertermia \\
Pulmonary actinomycosis & $1(3.1)$ & Histology & Organic LGF \\
Bacterial endocarditis & $2(6.2)$ & Transesophageal echocardiography & Organic LGF \\
Undiagnosed LGF & $4(12.5)$ & * & Organic LGF \\
Systemic lupus erythematosus & $1(3.2)$ & Autoantibodies & Organic LGF \\
Crohn's disease & $2(6.2)$ & Histology & Organic LGF \\
Ehlers-Danlos syndrome with diverticulitis & $1(3.1)$ & Colonoscopy & Organic LGF \\
Rheumatoid arthritis & $1(3.1)$ & Signs and symptoms/rheumatoid factor & Organic LGF \\
Food intolerance with bacterial overgrowth & $1(3.1)$ & Breath test & Habitual hypertermia \\
Dental granuloma & $1(3.1)$ & Orthopantomography & Habitual hypertermia \\
Appendicitis & $1(3.1)$ & Surgery & Habitual hypertermia \\
NH lymphoma & $1(3.1)$ & Histology & Organic LGF \\
\hline
\end{tabular}

$27 \pm 9.2$ years, $\mathrm{p}<0.02)$. Within group $\mathrm{B}$, age was significantly higher in men than women $(31 \pm 8.5$ vs. $24 \pm 5.5$ years, $\mathrm{p}<0.05)$; this difference was also present in group $\mathrm{A}$, but it was not significant ( $36.7 \pm 14$ vs. $31.7 \pm 14$ years; $\mathrm{p}=\mathrm{ns})$.

Table 2 shows the symptoms referred by the two groups. Dizziness and a not well-defined general malaise were the symptoms significantly associated in group B. Loss of weight, on the contrary, was

Table 2 Referred symptoms of patients with organic LGF (group A) and habitual hyperthermia (group B)

\begin{tabular}{llll}
\hline Symptoms & $\begin{array}{l}\text { Group A } \\
\boldsymbol{n}=\mathbf{3 2}\end{array}$ & $\begin{array}{c}\text { Group B } \\
\boldsymbol{n}=\mathbf{4 1}\end{array}$ & $\mathbf{p}$ \\
\hline Nausea & 1 & 5 & $\mathrm{~ns}$ \\
Anorexia & 1 & 5 & $\mathrm{~ns}$ \\
Arthralgia & 8 & 4 & $\mathrm{~ns}$ \\
Headache & 5 & 2 & $\mathrm{~ns}$ \\
Cough & 3 & 2 & $\mathrm{~ns}$ \\
Chills & 3 & 0 & $\mathrm{~ns}$ \\
Asthenia & 3 & 6 & $\mathrm{~ns}$ \\
Abdominal pain & 5 & 4 & $\mathrm{~ns}$ \\
Sore throat & 4 & 3 & $\mathrm{~ns}$ \\
Sweating & 3 & 0 & $\mathrm{~ns}$ \\
Dizziness & 0 & 7 & 0.02 \\
Pruritus & 0 & 2 & ns \\
Intercostal pain & 0 & 2 & ns \\
Not defined general malaise & 0 & 15 & 0.0001 \\
Weight loss & 9 & 1 & 0.05 \\
\hline
\end{tabular}

significantly associated to organic LGF (group A) $(\mathrm{p}<0.05)$.

Table 3 shows the anomalies found at physical examination. Lack of any pathological signs at physical examination was more frequent in subjects with $\mathrm{HH}(\mathrm{p}<0.0001)$, while the rate of splenomegaly and weight loss were significantly greater in group A ( $\mathrm{p}<0.03$ and $\mathrm{p}<0.05$ respectively).

Among the biochemical tests, white blood cells and CRP showed a higher number of elevated values in group A than in group B $[10 / 32$ vs. $1 / 41$ $(\mathrm{p}<0.05)$ and $9 / 32$ vs. $1 / 41 \quad(\mathrm{p}<0.05)$ respectively]. No difference was found between the two

Table 3 Presence of signs at physical examination in the two groups of patients

\begin{tabular}{lccc}
\hline Signs & $\begin{array}{c}\text { Group A } \\
\boldsymbol{n}=\mathbf{3 2}\end{array}$ & $\begin{array}{c}\text { Group B } \\
\boldsymbol{n}=\mathbf{4 1}\end{array}$ & $\mathbf{p}$ \\
\hline No signs & 2 & 30 & 0.0001 \\
Lymphadenopathy & 14 & 9 & $\mathrm{~ns}$ \\
Hepatomegaly & 3 & 4 & $\mathrm{~ns}$ \\
Splenomegaly & 6 & 0 & 0.03 \\
Pharyngeal inflammation & 3 & 0 & $\mathrm{~ns}$ \\
Skin lesions & 1 & 0 & $\mathrm{~ns}$ \\
Cardiac murmur & 3 & 0 & $\mathrm{~ns}$ \\
Pain on abdomen & 8 & 4 & $\mathrm{~ns}$ \\
$\quad$ palpation & & & \\
\hline Group A: organic LGF and group B: habitual hyperthermia.
\end{tabular}


groups for erythrocyte sedimentation rate (ESR) and urine analysis. None of the 41 subjects with a final diagnosis of $\mathrm{HH}$, however, showed particular alterations in biochemical parameters in the follow-up period; therefore, the alterations found in two patients at commencement of the study could have been because of seasonal viruses.

Thirty-two of the 41 subjects with $\mathrm{HH}$ who completed the 24 months of follow up had stopped measuring their body temperatures and defined their general health condition as good. At the first step, our diagnostic evaluation incorrectly classified four subjects as $\mathrm{HH}$ (false positive), thus having 91\% sensitivity and $87 \%$ specificity. After 6 months, diagnosis of $\mathrm{HH}$ was hypothesised in 42 patients, with only

Table 4 Discrimination of habitual hyperthermia from organic low-grade fever (LGF) at first evaluation (panel A) and after 6 months of follow up (panel B)

\begin{tabular}{llc}
\hline & $\begin{array}{l}\text { Habitual } \\
\text { hyperthermia }\end{array}$ & $\begin{array}{l}\text { Organic } \\
\text { LGF }\end{array}$ \\
\hline $\begin{array}{l}\text { Panel A } \\
\text { Correct diagnosis }\end{array}$ & 41 & 28 \\
Incorrect diagnosis & 4 & 0 \\
Panel B & 41 & 31 \\
Correct diagnosis & 1 & 0 \\
Incorrect diagnosis & & \\
\hline $\begin{array}{l}\text { Sensitivity 97\%; specificity 97\%; positive post-test probability } \\
97 \% \text {, sensitivity 91\%; specificity 88\%. }\end{array}$
\end{tabular}

one patient still incorrectly labelled as $\mathrm{HH}$, therefore with $97 \%$ sensitivity and specificity and 97\% posttest probability of $\mathrm{HH}$ (Table 4). Consequently, on the basis of these results, we propose a diagnostic algorithm (Figure 1), which is easy to apply and is also highly efficient for outpatients, but obviously needs to be confirmed by further studies.

\section{Discussion}

Fever of unknown origin is a well-defined entity with three classical diagnostic criteria, as first established by Petersdorf and Beeson: (i) illness of more than 3 weeks' duration, (ii) with a temperature higher than $38.3^{\circ} \mathrm{C}$ on several occasions and (iii) no established diagnosis after 1 week of evaluation (4). Thirty years later, Durack and Street proposed a reduction in the third criterion from 1 week to 3 days of appropriate investigations, because of the progress in diagnostic techniques (12). The other two criteria have remained unchanged. However, the second criterion (fever $>38.3^{\circ} \mathrm{C}$ ) excludes a series of fevers below $38.3{ }^{\circ} \mathrm{C}$, which must nevertheless be recognised to avoid discomfort to patients and prevent waste of public money, as they present a clinical course and pose diagnostic difficulties similar to those of FUO. In this report, we refer our experience of patients suffering from fever below $38.3^{\circ} \mathrm{C}$ and propose an algorithm to facilitate a correct diagnosis.

Our results, in contrast with the few epidemiological data in the literature $(4,5,12)$, show that the prevalence of $\mathrm{HH}$ is higher (54\%) than LGF from

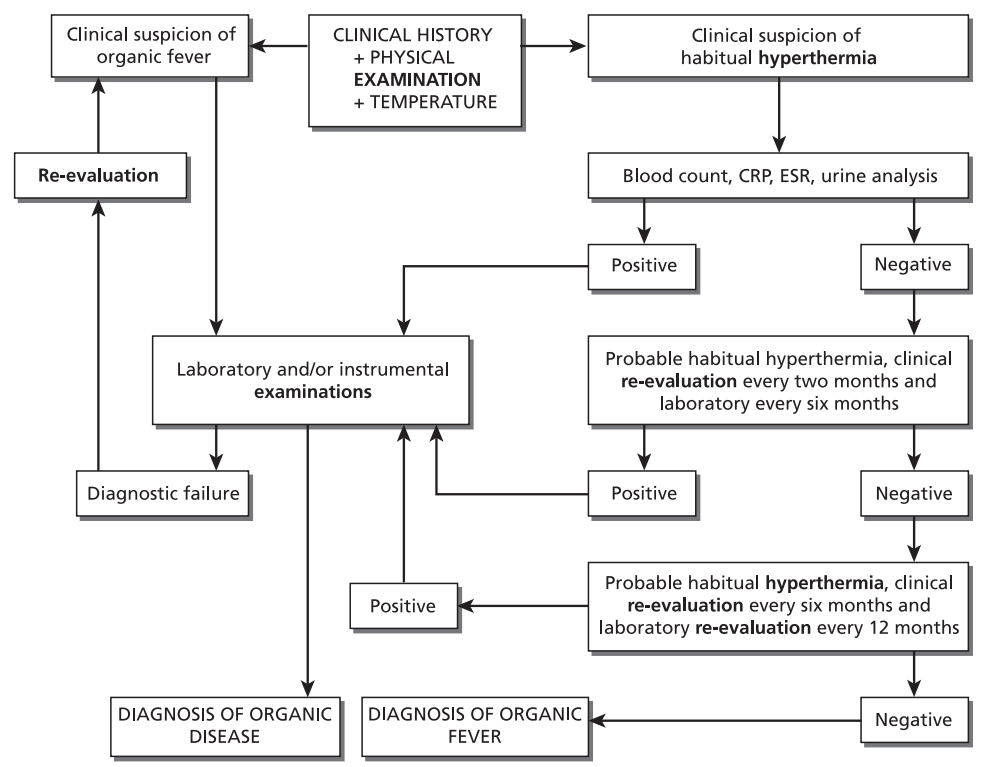

Figure 1 Algorithm for the diagnostic definition of low-grade fever 
organic causes $(46 \%)$. In the work of Knockaert et al. the prevalence of $\mathrm{HH}$ among $\mathrm{FUO}$ was as low as $2.5 \%$ (13). There may be various reasons for this difference:

- It could be because of a selection bias. Ours is a tertiary referral centre for fever pathologies and we are probably asked to investigate fevers for which no solution has been found in spite of the patient being followed for a long time by general practitioners or in non-specialised centres. HH may therefore be more difficult to evaluate than organic LGF;

- In the literature, $\mathrm{HH}$ is not always correctly defined and it is often mistakenly included with FUO, which, by definition, refers to patients with a body temperature $\geq 38.3{ }^{\circ} \mathrm{C}$, while $\mathrm{HH} \geq 38.3^{\circ} \mathrm{C}$ is an exceptional event. In this category of patients, $\mathrm{HH}$ may thus have only a marginal epidemiological role.

In our study, the $\mathrm{HH}$ subjects were prevalently woman, although this result was not statistically significant. Little is known about the causes of this higher frequency, already reported in the literature (6). Clinical history in $\mathrm{HH}$, in contrast with organic LGF, shows little of note: there is a prevalent symptomatology of cenesthopathy, dizziness and a not well-defined state of general malaise, which may also be correlated to a state of anxiety or depression as a consequence of the hyperthermia of unclear origin, as reported by Reimann (14). In contrast, weight loss is significantly associated with the organic forms. In this respect, it should be remembered that the above psychological states sometimes associated with $\mathrm{HH}$ should be investigated and evaluated because they could also lead to weight loss. In these cases, it may be useful to investigate for signs of weight loss and malnutrition during physical examination and in the laboratory tests.

Physical examination in our study population was negative in $73 \%$ of the $\mathrm{HH}$ patients compared with $6.2 \%$ in organic LGF, and the few clinical signs present in $\mathrm{HH}$ (for example lymphadenopathy and hepatomegaly), may be aspecific and misleading. Finally, simple and easily performable laboratory assays, such as total blood cell count and CRP were altered, with a higher frequency in organic LGF.

It should also be underlined that, as happens in FUO, a certain percentage of forms remain undiagnosed in LGF, in our study population $12.5 \%$. These forms can be distinguished by the initial presence of laboratory alterations, which however, rapidly normalise with the disappearance of the LGF. Precisely, this difference in temperature evolution - a normalisation in organic LGF with unknown aetiology compared with a persistent fever in $\mathrm{HH}$ - suggests that $\mathrm{HH}$ in LGF may be simply interpreted as an adjustment of the hypothalamus set-point towards higher values, and therefore considered a paraphysiological variant rather than a pathological condition. It may simply be one of the two extremities of the Gaussian distribution of human body temperature: the higher one.

In the light of the data we obtained, it is important to underline two aspects:

- a prolonged follow up, as mentioned above, represents the only valid diagnostic tool to exclude organic pathologies in patients with LGF;

- the diagnostic approach of our study, from which we deduced the above-mentioned algorithm, presents a $97 \%$ probability of diagnosis of $\mathrm{HH}$ at 6 months. This would allow us to already reassure the patient during the first few visits. Only by doing so can we prevent the hypochondriac delirium, which leads a patient to become thermometer-dependent and to construct a host of more or less complex symptoms, building up a clinical picture, which at that point is difficult to diagnose and at times can only really be cured by a psychiatrist.

As regards organic LGF, the small number of cases in our population did not allow us to reach any definitive conclusions, and as mentioned above it is difficult to compare our results because of the very few reports in the literature. What emerges is that, as in the majority of cases of FUO, the most frequent cause was infection followed by miscellaneous causes. This would confirm the emerging role of inflammatory non-infectious disease, which is being more frequently reported in the various study populations (15-17). The very small number of neoplasm cases found is, on the contrary, at first sight, difficult to understand; this is probably as a result of the fact that these patients also presented with a serious and complex clinical picture with, apart from the LGF, alarm signals, which warranted hospitalisation rather than outpatient treatment.

In conclusion, the results of our study population suggest: (i) that $\mathrm{HH}$ in LGF patients is an important nosographical entity with a high prevalence, confirming the importance of diagnosing it by limiting the diagnostic approach to a thorough physical examination and few but specific laboratory tests, and (ii) that it is incorrect to make a clear and substantial difference between the management of LGF and FUO. In LGF, after the HH forms have been eliminated, diagnostic work-up should not be different to that of FUO because, in general, the causes of FUO can also be responsible for LGF. 


\section{Acknowledgement}

We thank Dr Carole Greenall, BA, for the revision of the English language.

\section{References}

1 Blosson DB, Salata RA. Febbre e sindromi febbrili. In: Andreoli TE, Carpenter CJ, Griggs RC, Benjamin IJ, eds. Cecil Essentials of Medicine, 7th edn. Salerno: Saunders Elsevier, 2007: 775-87.

2 Takahashi A, Ishimaru H, Ikarashi Y, Kishi E, Maruyama Y. Hypothalamic neuroactivity in specific processes and central regulation of body temperature and water intake. Brain Res Brain Res Protoc 2001; 8: 68-73.

3 Ishiwata T, Hasegawa H, Yasumatsu $\mathrm{M}$ et al. The role of preoptic area and anterior hypothalamus and median raphe nucleus on thermoregulatory system in freely moving rats. Neurosci Lett 2001; 22: $126-8$.

4 Petersdorf RG, Beeson PB. Fever of unexplained origin: report on 100 cases. Medicine 1961; 40: 1-30.

5 Vanderschueren S, Knockaert D, Adriaenssens T et al. From prolonged febrile illness to fever of unknown origin: the challenge continues. Arch Intern Med 2003; 163: 1033-41.

6 Iandolo C, Manfellotto D. Patogenesi e significato biologico e clinico della febbre. Edizioni Luigi Pozzi. Le Febbri di Origine Oscura, una Guida Alla Diagnosi. Roma, 1987: 5-23.

7 Musher DM, Fainstein V, Young EJ, Pruett TL. Fever patterns. Their lack of clinical significance. Arch Intern Med 1979; 139: $1225-8$.
8 Mansueto S, Affronti M, Manfellotto D et al. Le Febbri di Origine Sconosciuta: un frequente, costoso cimento. Atti del $105^{\circ}$ Congresso Nazionale della Società Italiana di Medicina Interna, Palermo 2004; 19 (Suppl. 1): 125S-46S.

9 De Kleijn EM, Vandenbroucke JP, van der Meer JW. Fever of unknown origin. I A prospective multicenter study of 167 patients with FUO, using fixed epidemiologic entry criteria. The Netherlands Study Group. Medicine (Baltimore) 1997; 76: 392-400.

10 Arnow PM, Flaherty JP. Fever Of unknown origin. The Lancet 1997; 350: 575-80.

11 Zenone T. Fever of unknown origin in adults: evaluation of 144 cases in a non-university hospital. Scand J Infect Dis 2006; 38: 632-8.

12 Durack DT, Street AC. Fever of unknown origin - re-examined and redefined. Curr Clin Trop Infect Dis 1991; 11: 35-51.

13 Knockaert DC, Vanneste LJ, Vanneste SB, Bobbaers HJ. Fever of unknown origin in the 1980s. An update of the diagnostic spectrum. Arch Int Med 1992; 152: 51-5.

14 Reimann HA. Habitual hypertermia. JAMA 1932; 99: 1860.

15 Mansueto P, Di Lorenzo G, Rizzo M et al. Fever of unknown origin in a Mediterranean survey from a division of Internal Medicine: report of 91 cases during a 12-year-period (1991-2002) Intern Emerg Med 2008; 3: 219-26.

16 Barbado FJ, Vazquez JJ, Pena JM, Arnalich F, Ortiz-Vazquez J. Pyrexia of unknown origin: changing spectrum of diseases in two consecutive series. Postgrad Med J 1992; 68: 884-7.

17 Gaeta GB, Fusco FM, Nardiello S. Fever of unknown origin: a systematic review of the literature for 1995-2004. Nucl Med Comm 2006; 27: 205-11.

Paper received July 2009, accepted October 2009 